practice. The sample was limited to inpatients aged over 45 and admitted in 1981, whose illnesses placed them in 12 diagnosis related groups that met objective criteria related to throughput and mortality (the latter being important as one way of measuring differences in outcome). The purpose of the study was to assess differences in cost between the two services and to compare outcomes. Besides comparing the total cases in each diagnosis related group the investigators also selected within the sample 51 matched pairs for closer comparative analysis. Since the study took place in a single institution, prices and "facility" costs were held constant. Any cost differences thus stemmed from differences in physician behaviour.

The results showed higher costs on the faculty service in nine of the 12 diagnosis related groups. On average, after adjusting for case mix and socioeconomic characteristics, the cost differential for all 12 diagnosis related groups was around $10 \%$. Interestingly, the cost difference was smallest for the least seriously ill patients and largest for the most seriously ill - those who, at the time of admission, had an estimated probability of death of 0.25 or greater. Among these patients those treated on the faculty service had costs that were $70 \%$ higher than on the community service. The greatest differences lay in diagnostic costs. So far, one might say, there are no surprises.

To turn to outcomes, patients on the faculty service had better results, as measured by deaths per 100 admissions, in 11 of the 12 groups. After adjustments for case mix and socioeconomic characteristics, patients on the community service were one third more likely to be dead at discharge. In the series of matched pairs the death rate was about twice as high among patients on the community service.

On first view these results appear not only to document once again the higher costs of the teaching hospitals but also to vindicate them, so far as they stem from clinical decisions. Yet the matter is not as simple as that. To set aside any questions of methodology and replicability, there remain (as the researchers recognise) substantial uncertainties about the policy conclusions to be drawn. Most obviously, the large mortality difference at discharge on the two services had, in the series of matched pairs, vanished within 12 months of discharge. Could it be that substantial faculty efforts and expenditures in keeping very sick people alive had resulted only in buying quite temporary, evanescent benefits? Intriguingly, also, the balance of cost and outcome differences varied sharply by diagnosis. In one set of diagnosis related groups the faculty service showed substantially lower mortality at virtually no difference in cost. In a second set there were large differentials in both costs and benefits. And in a third there was a large cost differential, but death rates were similar on the two services. Particularly fascinating, and perhaps also amusing and reassuring, is the suggestion that patients or their referring physicians seemed to know a good deal about all this already, for the faculty service was most used where its results were best and its costs little different. When the reverse was the case the bulk of admissions flowed to the community service. To quote the researchers "It is as if most of the patients were assigned to the service that would provide the best balance of costs and benefits. Neither the faculty nor the community medical practice was necessarily better or worse, merely different."

That is a pleasing footnote to an elegant piece of research. One wonders whether it applies in the National Health Service. It links to another relevant conclusion-namely, that if the skill and effort of the teaching centres do indeed help some patients, then it becomes crucially important to identify those most likely to benefit from them.

Finally, research like this is refreshing at the present time in both the United States and Britain because it recognises that cost differences are only part (and ultimately the less important part) of the conundrum. Results, in terms of benefits to patients, matter even more.

ROBERT J MAXWELL

Secretary,

King Edward's Hospital Fund for London,

London W2 4HT

1 Department of Health and Social Security. Sharing resources for health in England: report of the Resource Allocation Working Pary. London: HMSO, 1976.

2 Feldstein MS. Economic analysis for health service efficiency. Amsterdam: North-Holland Publishing Company, 1967.

English T. The UK cardiac surgical register. In: Output measurement for health services. London: CASPE Research Project, King Edward's Hospital Fund, 1982.

4 Flood AB, Scott WR, Ewy W. Does practice make perfect? Parts 1 and 2. Med Care 1984;22:

Garber AM, Fuchs VR, Silverman JF. Case mix, costs and outcomes. $N$ Engl f Med 1984;310:1231.

\section{Delayed deterioration of consciousness after trivial head injury in childhood}

Minutes to hours after an apparently minimal head injury a child may show a delayed deterioration of level of consciousness. In a recent study by Snoek et al this late deterioration occurred in $4 \%$ of children with head injuries; of the 42 affected, three died. ${ }^{1}$ Unfortunately, no necropsies were carried out. The authors questioned whether the admission policy after head injury in childhood requires revision based on these data.

What are the possible pathophysiological causes of such secondary deterioration, the expected outcome, and possibilities for medical intervention? There is no single pathological cause. The first concern when the history of trauma appears to be at odds with the clinical picture should be that the history is false. In the United States child abuse accounts for roughly 2000 childhood deaths a year and there are some 2 million reported cases. When the child's custodian is responsible for the trauma, fear and guilt may delay seeking medical care and result in the fabrication of a story-which usually includes an acute episode of change of consciousness after a trivial insult. Finding retinal haemorrhages in a child under 1 year old is almost pathognomonic of the "shaking injury." In older children no such clues are available. Nevertheless, when the history conflicts with the clinical findings child abuse must be suspected and that suspicion followed up to ensure that such trauma is prevented from happening again.

The second cause of acute alteration in consciousness after minor trauma is a seizure; this probably explains about half of all cases of acute delayed deterioration. Usually there is no history of seizures. In general, symptomatic support of the child after the seizure is all that is required, and anticonvulsant drugs are rarely needed.

Next, secondary deterioration of consciousness must always arouse suspicion of a mass lesion. In children this is uncommon, and only 5\% of chidren with Glasgow coma scores of 8 or less will have an epidural haematoma; but $16 \%$ of children under 2 years admitted to our neurosurgical service have an epidural haematoma. The presence of a 
surgically treatable mass is best excluded by computed tomography. Furthermore, a small epidural haematoma may present with an acute deterioration of consciousness 24-48 hours after injury in a child whose clinical course has been progressive improvement. In these children the main problem is diffuse brain swelling rather than the volume of the haematoma.

The final cause of acute worsening after trivial head injury is the so called "paediatric concussion syndrome," seen very frequently in the child under 1 year. Minutes to an hour or more after a minimal head injury the child becomes pale, sweating, irritable, and sleepy and may vomit. These features may be precipitated by a second minor fall. The clinical picture is similar to an acute vasovagal attack. The fontanelle, if open, is usually soft. This is a self limiting syndrome and no treatment is needed (other than fluid support). I have never seen a child in this age group become unconscious or die as a result of this syndrome. If the fontanelle is full a mass lesion must be ruled out, and computed tomography is the best test. The most frequent clinical accompaniment of this syndrome is truncal ataxia.

In children over 1 year old a similar syndrome occurs but may progress to coma with pupillary changes, apnoea, and occasionally death. The most frequent signs are in the pupils, with alternating dilatation, constriction, hippus, or absence of response to light. The coma may be severe enough for ventilation to be required. I have never seen this syndrome result in death, but such deaths are well documented. What necropsy evidence there is shows minimal or no primary cerebral damage, diffuse swelling of the brain, and congestion of small blood vessels, especially in the white matter.

The initial treatment for children progressing to coma is endotracheal intubation and hyperventilation in an effort to alleviate any cerebral congestion, together with monitoring and treatment of raised intracranial pressure. Computed tomography most frequently shows small ventricles and partial or complete obliteration of the subarachnoid spaces around the brain stem. Cerebral blood flow studies have shown relatively increased flow, and recently measurements of cerebral metabolic rate for oxygen have shown increased values in this group of children with diffuse swelling, despite low Glasgow coma scores. Thus vasodilatation seems to occur in the brain early in the course of the syndrome of secondary deterioration. What is not clear is whether the vasodilatation is the result of increased metabolic activity or not, and whether there is sufficient vasodilatation to produce intracranial hypertension, herniation, and death.

Possibly the self limiting syndrome of the small child and the more dramatic, but usually reversible, syndrome in the older child have a common aetiology. The delayed onset suggests either delayed effects of mechanical trauma, as shown by Ward et al, or some chemical mediator that must percolate through the brain and ventricular system until it enters the periaqueductal grey matter at the region of the fourth ventricle. ${ }^{2}$ Stimulation of the vagal nucleus and periaqueductal grey reticular formation would account for the vomiting and drowsiness. Furthermore, stimulation in this region produces vasodilatation in the cortex with or without increased metabolic activity. If this explanation is correct the vasovagal syndrome would then be the least manifestation of this brain stem stimulation; in small children the presence of an open fontanelle and relatively large cerebrospinal fluid spaces will prevent a sudden rise in intracranial pressure, and so the occurrence of apnoea and death. In children with closed fontanelles excitation of the floor of the fourth ventricle and periaqueductal grey matter accounts for the increased cerebral metabolic activity, the increase in cerebral blood flow and volume, and the signs of alterations of pupillary function and deterioration of consciousness. In a few children the vasodilatation-possibly accompanied by brain oedema-will cause a sudden rise in intracranial pressure, compression of the medulla, apnoea, and death.

This is one hypothesis, but we have insufficient evidence to define the pathophysiology of this syndrome. Whatever the underlying cause, the syndrome of acute deterioration after trivial head trauma in children is real. Child abuse, an intracranial haematoma, or a seizure must be considered by the physician caring for the child. I do not believe that the frequency of the paediatric concussion syndrome or malignant brain swelling is such that any change is needed on policies for admission after head injury. Any such child showing disturbed consciousness or focal neurological deficit requires admission to hospital. Physicians caring for children must, however, be aware that this syndrome exists, and must educate the parents so that they will return to the accident and emergency department immediately should any such change occur. Any child showing features of this syndrome requires close observation in hospital, and in the rare case where coma ensues endotracheal intubation and hyperventilation should be started at once. Monitoring of intracranial pressure and treatment of any rise will also ensure that recovery is as complete as possible.

\section{DeREK A Bruce}

Associate Professor of Neurosurgery and Paediatrics,

Unuversity of Pennsylvania School of Medicine, and

Associate Neurosurgeon,

Children's Hospital of Philadelphia,

Philadelphia,

Pennsylvania 19104

USA

1 Snoek JW, Minderhoud JM, Wilmink JT. Delayed deterioration following mild head injury in children. Brain 1984;107:15-36

2 Foltz EL, Jenkner FL, Ward AA Jr. Experimental cerebral concussion. I Neurosurg 1953;10:342- 